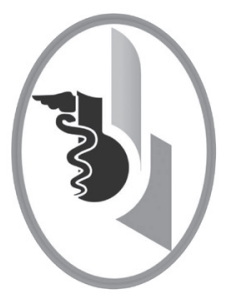

\title{
ANOMALÍAS CONGÉNITAS EN FAMILIAS DE ARARCA (ISLA DE BARÚ). BOLÍVAR-COLOMBIA
}

\author{
CONGENITAL ABNORMALITIES IN FAMILIES FROM ARARCA \\ (BARÚ ISLAND)
}

\author{
Espitia-Almeida Fabián ${ }^{1}$ \\ Ospina-Mateus Laura² \\ Mora-García Gustavo ${ }^{3}$ \\ Gómez Camargo Doris ${ }^{4}$ \\ Malambo-García Dacia ${ }^{5}$
}

Correspondencia: dmalambog@unicartagena.edu.co

Recibido para evaluación: febrero - 15 - 2015. Aceptado para publicación: octubre - 25 - 2015.

\section{RESUMEN}

Introducción: las enfermedades genéticas y los defectos de nacimiento son considerados problemas de salud pública a nivel mundial, con una incidencia de alrededor del $1 \%$. Las principales complicaciones clínicas de estas enfermedades se caracterizan por comprometer la calidad de vida de los afectados, causando una grave discapacidad intelectual o física, por su carácter progresivo y condicionan una mortalidad precoz de los individuos afectados y generan una carga en las familias de las personas que las padecen.

Objetivo: caracterizar las familias que reporten personas con anomalías congénitas que se puedan catalogar como genéticas, incluyendo el componente socioeconómico.

Materiales y métodos: se planteó una metodología descriptiva, observacional y se analizaron algunos aspectos genéticos, como historia clínica genética y citogenética; se incluyeron variables socioeconómicas de una comunidad rural que previamente evidenció la presentación de enfermedades genéticas.

Resultados: se reportaron cinco casos de personas con enfermedades congénitas y con indicación de trastorno genético, tres casos pertenecían a una misma familia; se analizó el componente sociodemográfico. Se evidenció un estado de pobreza de las familias, nivel educativo bajo en la mayoría, y casos de analfabetismo. Salarios que no alcanzan el mínimo, hacinamiento de las personas en las viviendas y relaciones intrafamiliares quebradas, evidente apatía entre los integrantes de las familias estudiadas.

Conclusión: esta investigación permitió verificar en la comunidad de Ararca cinco casos de trastornos genéticos que podrían estar asociados a compromiso con factores ambientales y socioeconómicos. Se recomienda brindar apoyo interdisciplinario a las comunidades y trabajar en el diagnóstico a temprana edad con el fin de evitar discapacidad para desempeñarse en la sociedad y mitigar el impacto sobre las familias afectadas. Rev.cienc.biomed. 2015;6(2):251-258

\section{PALABRAS CLAVE}

Anomalías congénitas; Herencia; Salud pública; Relaciones familiares.

\footnotetext{
Químico, Estudiante de Maestría en Bioquímica. Joven Investigador COLCIENCIAS, Grupo de Investigación UNIMOL, Universidad de Cartagena. Bacterióloga, Joven Investigador COLCIENCIAS, Grupo de Investigación UNIMOL. Universidad de Cartagena.

Médico. Doctorando en Medicina Tropical, Facultad de Medicina. Grupo de Investigación UNIMOL. Universidad de Cartagena.

4 Bacterióloga, MSc. Ph. D. Facultad de Medicina. Directora Regional Doctorado en Medicina Tropical SUE Caribe. Grupo Investigación UNIMOL. Universidad de Cartagena

4 Bióloga, MSc. Genética Humana. Docente Universidad de Cartagena, Facultad de Medicina. Grupo de Investigación UNIMOL, Universidad de Cartagena.
} 


\section{SUMMARY}

Introduction: genetic diseases and birth defects are considered problems of public global health, they have $1 \%$ incidence in this problem. The main clinical complications of these diseases are characterized by compromising the quality of life of those affected, causing a severe disability mental or physical, for its progressive and conditional early mortality in these affected individuals. They also generate a burden on families of people who have them.

Objective: to classify the families to report people with congenital diseases that can be classified as genetic diseases also including socio-demographic aspects.

Material and method: a descriptive, observational methodology was proposed and some genetic aspects such as medical history and genetic analysis of chromosomes analysed. Some socioeconomic variables in a rural community that previously showed the presentation of genetic diseases were analyzed.

Results: five cases of people with congenital diseases indicating genetic disorder were found, including three cases belonging to the same family; the socio-demographic component showed poverty families, low educational levels and in most cases illiteracy is analyzed. Wages do not reach the average, overcrowding of people in homes and broken family relationships and apathy among members of the families studied.

Conclusion: it was possible to verify five cases of genetic disorders that may be associated with commitment to environmental and socioeconomic factors; it is recommended to provide interdisciplinary support to communities and work in the diagnosis at an early age in order to prevent disability to function in society and mitigate the impact on affected families. Rev.cienc.biomed. 2015;6(2):251-258

\section{KEYWORDS}

Congenital Abnomalities; Heredity; Public health; Family relations.

\section{INTRODUCCIÓN}

Enmarcado en la estrategia de reducción de los riesgos de contaminación de los ecosistemas relacionados con la Bahía de Cartagena, Canal del Dique e Islas del Rosario y Barú se analiza la iniciativa de los problemas de salud que afectan a la comunidad de Ararca desde el componente genético o hereditario que pudiese estar relacionado.

Las enfermedades genéticas y los defectos de nacimiento son considerados problemas de salud pública a nivel mundial, en países latinoamericanos se manifiesta como una deficiencia en los servicios de apoyo a las enfermedades genéticas $(1,2)$. Estas patologías constituyen un grupo muy importante, no solo por su incidencia relativamente elevada (alrededor de $1 \%$ de los bebes nacen con algún tipo de anormalidad genética), sino también por el tipo de problemas que producen $(3,4)$. Desde el punto de vista clínico estas enfermedades se caracterizan por comprometer la calidad de vida de los afectados, causando una grave discapacidad intelectual o física. Así mismo, es frecuente que estas enfermedades tengan un carácter progresivo y condicionen una mortalidad precoz en los individuos afectados $(3,5)$.
Se evidencia una carga potencial de las enfermedades genéticas que plantea la necesidad de implementar más centros para el entrenamiento de personal con especialistas en genética que puedan en el futuro brindar servicios adecuados de diagnóstico y asesoría genética (6). Es necesario realizar diagnósticos a temprana edad, campo donde las técnicas moleculares y de citogenética juegan un papel central para la correcta caracterización de las enfermedades, así como para establecer tratamientos farmacológicos adecuados $(3,4)$.

En Colombia las enfermedades genéticas tienen una frecuencia de alrededor 37.3 por cada 1000 habitantes, mostrando un impacto notable y negativo sobre una proporción de la población $(6,7)$. En esta cifra se incluyen las malformaciones congénitas que corresponden al $50 \%$ del total de las patologías estudiadas (6). En Cartagena se han publicado resultados sobre algunas enfermedades de origen genético (8) que muestran el efecto social generado en las familias afectadas y cómo esto puede ser excluyente ante la sociedad.

Un estudio socioeconómico y demográfico realizado en la Isla de Barú, dirigido a las co- 
ISSN: 2215-7840, 6(2), julio-diciembre 2015, Espitia-Almeida Fabián, Ospina-Mateus Laura, Mora-García Gustavo,

Gómez Camargo Doris, Malambo-García Dacia

munidades de Santa Ana muestra que existe un mayor riesgo de padecer enfermedades de origen genético y no genético a causa de las condiciones precarias en las que habitan (9). Asimismo, se encontraron dos estudios sobre la caracterización sociodemográfica de las comunidades de la isla, entre ellas la comunidad de Ararca, reportando un gran índice de pobreza: el 53\% de sus habitantes generan ingresos inferiores al salario mínimo, no cuentan con alcantarillado, ni servicio telefónico, el $40 \%$ de la población no sabe leer ni escribir y menos del $1 \%$ de la población ha realizado estudios superiores (10).

En cuanto a los servicios de salud, es una comunidad que cuenta con mayores deficiencias debido a la usencia de un centro de atención en salud, por tanto, deben desplazarse a la comunidad de Santa Ana para recibir atención de primer nivel, y para recibir servicios más complejos deben desplazarse al distrito de Cartagena (11).

Dado lo anterior, se pretende realizar una caracterización de las familias que reporten personas con enfermedades congénitas que se puedan catalogar como genéticas junto con el componente socioeconómico.

\section{MATERIALES Y MÉTODOS}

\section{Tipo de estudio}

El tipo de estudio utilizado en esta caracterización de las enfermedades genéticas en la comunidad de Ararca fue descriptivo-explorativo y de corte transversal, debido a que se observaron, detallaron, interpretaron y analizaron variables socioeconómicas y genéticas de una comunidad poco estudiada, en un tiempo fijo y único.

\section{Ubicación geográfica y descripción de la población}

Ararca está situada en la costa del Caribe colombiana, muy cerca del casco urbano de la ciudad de Cartagena, a una distancia aproximada de 55 kilómetros, específicamente en la Isla de Barú; la cual se ubica en el archipiélago de las Islas del Rosario, Parque Nacional Natural (Decreto 0977 del 2001) que tiene una extensión aproximada de 7 hectáreas.
Esta población tiene alrededor de 1.047 a 1.200 habitantes (10). Con una distribución por sexo de $47.85 \%$ hombres y $52.15 \%$ mujeres y una pirámide poblacional distribuida por edad que muestra una mayor proporción de la población con edades de 25-55 años $(38.54 \%)$, edad productiva y reproductiva según las encuestas realizadas por la Fundación Mamonal en el año 2009. Además presenta una población entre 1-5 años del $8.02 \%$, una población mayor de 55 años del $11.30 \%$, una fracción del $14.99 \%$ con edades entre 6-11 años, $12.51 \%$ para la población de edades entre $12-15$ y para la población de 17-24 años una proporción de 14.61\% (10).

\section{Antecedentes de investigación en Ararca}

Previo al presente reporte, los investigadores responsables del componente Índices de Salud del proyecto BASIC, observaron en la comunidad de Ararca a residentes con manifestaciones fenotípicas de malformaciones congénitas. Los datos de nombres y direcciones se archivaron y retomaron para el análisis posterior representado en este informe de resultados.

\section{Muestreo y selección de pacientes}

El muestreo fue realizado por conveniencia, dirigido durante las salidas de campo a la comunidad de Ararca. Se indagó sobre personas que presentaran alguna anomalía al nacer como el principal criterio de inclusión. Las personas o cuidadores que accedieron a participar en el estudio firmaron el consentimiento informado. Se tomó una muestra de sangre para estudio de cromosomas con bandeamiento G, además, se realizó valoración médica con elaboración de árbol genealógico y se realizaron familiogramas.

\section{Aspectos sociodemográficos}

Para determinar los aspectos sociodemográficos de las familias se realizó una encuesta de familia denominada "Enfermería en la familia y en la comunidad" diseñada por la Facultad de Enfermería de la Universidad de Cartagena.

\section{Construcción de familiograma}

A los pacientes seleccionados se les construye un familiograma o genograma para vi- 
sualizar la estructura o composición familiar, para ello se realizó una encuesta sobre la estructura de la familia.

\section{Construcción de árbol genealógico}

Con el fin de determinar la posible transmisión hereditaria de las afecciones genéticas presentadas en las familias se elaboró el árbol genealógico siguiendo los símbolos estándar.

\section{Citogenética}

Se analizan los cromosomas de los pacientes en búsqueda de alteraciones que permitan relacionar estados de retraso mental, falla en el crecimiento o algunos defectos congénitos.

\section{RESULTADOS}

\section{Descripción de los casos}

En la Tabla $\mathrm{N}^{\circ} 1$ se presenta la descripción de las características físicas y clínicas de los cinco pacientes estudiados en la comunidad de Ararca (Isla de Barú). Se destacan malformaciones en el sistema musculoesquelético y retardo mental.

Las variables: sexo, edad, talla y peso halladas en los sujetos estudiados se describen en la Tabla $N^{\circ} 2$.

Los casos 3, 4 y 5 pertenecen a una misma familia, (casos 4 y 5 hermanas) y caso 3 prima de las dos anteriores.

\section{Información sociodemográfica}

Las viviendas presentan una buena condición en su estructura física referente a materiales que componen las paredes (construidas con block), pisos (de cemento lustrados) y material de techos en Eternit.

\begin{tabular}{|c|c|c|c|c|}
\hline \multicolumn{5}{|c|}{$\begin{array}{c}\text { TABLA No } 1 . \\
\text { DESCRIPCIÓN DE LOS CASOS ENCONTRADOS CON POSIBLES TRASTORNOS GENÉTICOS }\end{array}$} \\
\hline Caso & Género & $\begin{array}{l}\text { Edad } \\
\text { (años) }\end{array}$ & Etnia & Descripción \\
\hline 1 & M & 66 & Negra & $\begin{array}{l}\text { Sindactilia de ambas manos, afectados los dedos } 3,4 \text { y } 5 \text {. Latido } \\
\text { fácilmente observable y palpable, punto de máximo impulso a } \\
\text { nivel de apófisis xifoides. En epigastrio, los ruidos se escuchan } \\
\text { con máxima claridad. Paciente consciente, alerta, orientado en } \\
\text { las } 3 \text { esferas, fuerza en } 4 \text { extremidades } 5 / 5 \text {, no hay asimetría } \\
\text { facial, marcha normal, habla fluida y coherente. }\end{array}$ \\
\hline 2 & M & 23 & Negra & $\begin{array}{l}\text { Múltiples alteraciones motoras que impiden la bipedestación y } \\
\text { la marcha. Extremidades superiores hipertróficas e inferiores } \\
\text { atróficas. Desviación de pies hacia la línea media. Desviación } \\
\text { mandibular hacia la izquierda, dificultad para articular palabras, } \\
\text { flexión de la articulación del codo y de muñecas. Fuerza en } \\
\text { extremidades superiores } 5 / 5 \text {, en extremidades inferiores } 3 / 5 \text {. } \\
\text { Se moviliza arrastrando su cuerpo con la ayuda de sus brazos. } \\
\text { Comprende las preguntas que se le realizan y obedece órdenes. }\end{array}$ \\
\hline 3 & $F$ & 12 & Negra & $\begin{array}{l}\text { Retraso mental, ausencia de lenguaje, emite sonidos guturales, } \\
\text { afecto alegre (sonrisa constante no social), microcefalia, } \\
\text { fisuras palpebrales oblicuas con desviación mongoloide, boca } \\
\text { permanentemente abierta, macrostomía y babeo de saliva } \\
\text { constante, hiperplasia gingival, dientes separados y brazos } \\
\text { flexionados durante la marcha. }\end{array}$ \\
\hline 4 & $\mathrm{~F}$ & 8 & Negra & $\begin{array}{l}\text { Manifestaciones similares al caso } 3 \text {, retraso mental, ausencia del } \\
\text { lenguaje, afecto alegre, hiperactividad y excitabilidad marcada } \\
\text { con movimientos de aleteo en las manos ante los estímulos, } \\
\text { estrabismo divergente alternante, boca permanentemente } \\
\text { abierta, dientes separados y babeo constante de saliva. }\end{array}$ \\
\hline 5 & $F$ & 3 & Negra & $\begin{array}{l}\text { Manifestaciones similares a los casos } 3 \text { y } 4 \text {, siendo los trastornos } \\
\text { ligeramente menos evidentes. Ausencia de lenguaje, afecto } \\
\text { alegre, microcefalia ( } 44 \mathrm{~cm}) \text {, dientes separados, babeo de } \\
\text { saliva constante, hiperactividad y ataxia de la marcha. }\end{array}$ \\
\hline
\end{tabular}

M: Masculino; F: Femenino. 


\begin{tabular}{|c|c|c|c|c|}
\hline \multicolumn{5}{|c|}{ TABLA NO 2.} \\
VARIABLES DEMOGRÁFICAS DE LOS \\
PACIENTES ENCONTRADOS CON POSIBLES \\
TRASTORNOS GENÉTICOS \\
\hline Casos & Sexo & $\begin{array}{c}\text { Edad } \\
\text { (años) }\end{array}$ & $\begin{array}{c}\text { Peso } \\
(\mathrm{Kg})\end{array}$ & $\begin{array}{c}\text { Talla } \\
(\mathrm{mts})\end{array}$ \\
\hline $\mathbf{1}$ & $\mathrm{M}$ & 66 & 52 & 1.66 \\
\hline $\mathbf{2}^{*}$ & $\mathrm{M}$ & 23 & --- & --- \\
\hline $\mathbf{3}$ & $\mathrm{F}$ & 12 & 35.6 & 1.42 \\
\hline $\mathbf{4}$ & $\mathrm{F}$ & 8 & 22.4 & 1.21 \\
\hline $\mathbf{5}$ & $\mathrm{F}$ & 3 & 10.1 & 0.89 \\
\hline Promedio & --- & 22.4 & 30.02 & 1.29 \\
& & & ${ }^{* *}$ & \\
\hline
\end{tabular}

* A este paciente no se le pudo determinar el peso y talla por las forma como se ha curvado su cuerpo, se dificulta medición y pesaje en una balanza plana.

** Estos promedios no incluyen los datos del caso dos por lo antes mencionado.

Se observó hacinamiento en las viviendas, en las tres casas se encontraron dos habitaciones y el número de personas que conforman los hogares oscila entre cuatro a ocho, el caso más extremo fue la casa 3 con un promedio de cuatro personas por habitación. El hacinamiento, junto con los bajos ingresos económicos y el deficiente nivel educativo reflejan la pobreza en las que viven las personas afectadas y sus familiares (12). En la Tabla $N^{\circ} 3$ se describen estos aspectos.

Las variables socioeconómicas colectadas se reportan en la Tabla 4 . En ella se muestra información respecto al nivel educativo, actividades laborales, ingresos económicos y seguridad social.

\section{Familiogramas}

Los familiogramas mostraron que las familias presentan conflictos internos. La madre del paciente 2 lo cedió a la abuela materna para que ella lo cuidara. Se evidenció que las parejas no son estables y que mujeres y hombres tienen más de una pareja. También se encontró que las personas tienen relaciones sentimentales con sus familiares: primos, primas, tíos, tías.

\begin{tabular}{|l|c|c|c|}
\hline \multicolumn{4}{|c|}{ TABLA No 3.} \\
CONDICIÓN DE LAS VIVIENDAS DE CADA \\
FAMILIA ESTUDIADA
\end{tabular}

* El agua para consumo se almacenaba en tanques sin tapa según lo observado en el momento de la visita GN: gas natural

\begin{tabular}{|c|c|c|c|c|}
\hline \multicolumn{5}{|c|}{$\begin{array}{l}\text { TABLA NO } 4 . \\
\text { ALGUNAS VARIABLES SOCIOECONÓMICAS DE LAS FAMILIAS } \\
\text { ESTUDIADAS }\end{array}$} \\
\hline Nivel educativo & Casa 1 & Casa 2 & Casa 3 & Promedio \\
\hline Ninguno & 1 & 3 & 3 & 3.5 \\
\hline Primaria incompleta & 1 & 1 & 3 & 2.5 \\
\hline Primaria completa & 1 & 0 & 0 & 1 \\
\hline $\begin{array}{l}\text { Secundaria } \\
\text { incompleta }\end{array}$ & 0 & 0 & 2 & 2 \\
\hline Secundaria completa & 4 & 1 & 0 & 2.5 \\
\hline $\begin{array}{l}\text { Ingresos mensuales } \\
\text { promedio } \\
\text { (\$ Pesos } \\
\text { Colombianos) }\end{array}$ & 600000 & 600.000 & 200.000 & $466.666,67$ \\
\hline Seguridad social & \multicolumn{3}{|c|}{ Régimen subsidiado } & --- \\
\hline
\end{tabular}

Nota: Todas las familias estudiadas pertenecen al estrato 1, según la clasificación del SISBEN. 
Árboles genealógicos La Figura No 1 muestra el primer caso II4, donde el hermano II2 falleció por problemas cardíacos y presentó igual condición que el caso índice.

En la Figura No 2 se muestra al caso 2 representado por el segundo de cuatro hermanos, y según lo reportado por la familia no hay más miembros con esta condición.

En la Figura No 3 se representan los casos 3, 4 y 5, correspondientes a dos hermanas y una prima, todas con retardo mental y hallazgos clínicos similares.

\section{Cariotipos}

A los casos 1,2 y 4 se les realizó cariotipo con banda G. Los resultados para la resolución empleada fueron normales. El caso 4 tuvo un reducido índice mitótico.

\section{DISCUSIÓN}

El propósito del presente trabajo fue realizar una caracterización de las familias que reportaran personas afectadas con enfermedades expresadas desde el nacimiento que se pudieran catalogar como enfermedades genéticas. Se analizaron aspectos socioeconómicos, en lo que se encontró un alto índice de pobreza, con ingresos mensuales que oscilan entre 200.000-600.000 pesos colombianos (Tabla No 4), valores similares a los reportados por la Fundación Puerto Bahía en 2009 (10) y siguiendo los patrones regionales de pobreza que se reportan para Cartagena y Colombia (11).

Estas cifras no muestran un desarrollo para la ciudad y sus alrededores, a pesar de con- tar con aproximadamente 18.000 empresas reportadas por la Cámara de Comercio de Cartagena (11). Pobreza que incide en alto hacinamiento donde en una habitación duermen alrededor de tres personas, niños con deficiencia alimenticia, manifestada con bajo peso para la edad (13).

El nivel educativo de las familias estudiadas estuvo entre ningún nivel (las personas no han asistido nunca a un plantel educativo) y primaria incompleta (las personas asistieron a un plantel educativo, pero por motivos no expresados decidieron retirarse de la escuela) en mayor proporción, resultados simila- 
ISSN: 2215-7840, 6(2), julio-diciembre 2015, Espitia-Almeida Fabián, Ospina-Mateus Laura, Mora-García Gustavo,

Gómez Camargo Doris, Malambo-García Dacia

res a los reportados en el 2009 (10). Las familias atendidas reflejan la realidad que vive una comunidad olvidada, el panorama observado durante el recorrido por la comunidad en general es similar al de las estudiadas.

Los aspectos genéticos que podrían limitar las actividades de los pacientes y acarrear una carga en sus familias no se evidenció en la familia 1 , debido a que el sujeto realiza actividades laborales y cotidianas normales, asegurando no estar enfermo y ser el proveedor del sustento diario para su familia a pesar de la evidente sindactilia (14), que afirma no lo limita para hacer actividades físicas diarias (14-16), y no como en casos más severos en los que se compromete la movilidad completa de las manos o pies (17), y que como consecuencia puede causar rechazo por la sociedad $(7,18)$.

La evidente anormalidad cardíaca del caso 1 no fue explorada aunque se le recomendó acudir al servicio de salud lo más pronto posible debido al antecedente del hermano del paciente con afección similar y quien falleció por paro cardíaco. Para la sindactilia los factores genéticos se consideran la principal causa, adicionalmente podrían estar relacionados factores de contaminación ambiental; la situación económica desfavorable de la familia durante varias generaciones podría conllevar a un estado nutricional deficiente, constante y temprano durante el embarazo (19). Algunas personas habitantes de Ararca indican que en la población cercana, Barú, habitan personas con dedos adicionales en manos o pies (polidactilia) y otras personas con sordera. Se prevé verificar esos posibles casos en estudios futuros.

El caso 2 plantea dudas sobre la posible causa genética, pues es primera vez que se presenta en la familia según refieren los allegados. La madre indicó que diferente a sus otros hijos el paciente solo empezó el sostén cefálico hacia los tres meses de vida, lo que podría indicar que el sistema musculoesquelético estuvo comprometido desde el nacimiento.

Los casos 3, 4 y 5 de la tercera familia donde los pacientes son todas de sexo femenino podrían padecer una enfermedad genética relacionada con el cromosoma X (20-22), permitiendo que las manifestaciones sean predominantes en mujeres (21). Aunque es llamativa la expresión de sonrisa constante, aleteo de manos y locomoción similar a los pacientes con síndrome de Angelman. El bajo índice mitótico del caso 4, única muestra disponible de la familia 3 plantea dudas que no pudieron resolverse con la metodología utilizada.

En este estudio se observa en Figura 3 que la madre de los casos 4 (III6) y 5 (III8) concibió las dos niñas con padres diferentes; la niña del caso 5 es gemelo dicigótico, su hermano varón es normal. Mientras tanto, la prima de las anteriores, caso 3 (III1) presentó la enfermedad, cosa que evidenció que podría tratarse de una condición transmitida de madres a hijas. Para aclarar la causa genética de estos casos y los anteriores se requiere realizar estudios por biología molecular.

Finalmente, se muestra la necesidad de realizar este tipo de estudios en comunidades de la Isla de Barú, con el objetivo de caracterizar y detectar cualquier afección presentada que permita crear programas de promoción y prevención respecto a las enfermedades genéticas.

Se planteó como limitante el tamaño de la muestra, lo que sugiere promover futuras investigaciones con la comunidad con el objetivo de identificar personas afectadas que en esta primera búsqueda no fueron detectadas.

\section{CONCLUSIÓN}

Se evidenciaron cinco posibles casos de trastornos genéticos en la comunidad de Ararca (Isla de Barú) distribuidos en tres grupos familiares, de ellos tres casos pertenecen a una misma familia.

La pobreza de esta comunidad es evidente al observar el nivel educativo, los ingresos económicos y estilo de vida que los condiciona a vivir en hacinamiento (alrededor de tres personas por habitación). Este estudio inicial es una alerta para que los entes gubernamentales presten la debida atención a este tipo de problemas de salud en esta comunidad. 
Adicionalmente, en todo el recorrido realizado en la comunidad de Ararca se evidenció que no cuenta con un centro de servicios de salud debidamente dotado para el diagnóstico y atención oportuna de estas enfermedades de importancia para la salud pública.

\section{CONFLICTOS DE INTERESES: ninguno} que declarar.

FINANCIACIÓN : este trabajo hace parte del convenio "Manejo integrado de los recursos hídricos en la zona costera de CartagenaColombia: ciencia para tomadores de deci- sión y comunidades locales hacia la reducción de riesgos de contaminación y adaptación al cambio climático. (MIRH-Cartagena)"Interacciones entre cuencas, mar y comunidades (BASIC Cartagena), financiado por el International Development Research Centre (IDRC, Canadá).

\section{AGRADECIMIENTOS:}

A Yoled Vizcaíno López, médico joven e investigador quien colaboró con la revisión de los pacientes. A las personas de la comunidad de Ararca que se destacan por su amabilidad y colaboración.

\section{REFERENCIAS BIBLIOGRÁFICAS}

1. Zubieta-Ruiz B, Sánchez-Márquez P, Castillo-Cruz R. Enfermedades genéticas y defectos al nacimiento. Impacto en la morbilidad y mortalidad pediátrica. Acta Pediatrica de Mexico.2009;4:220-5.

2. Navarro EG. Genética clínica y dismorfología: generalidades. Revista española de pediatría: clínica e investigación. 2009;65(1):12-4.

3. González-Lamuño D, García Fuentes M. Enfermedades de base genética. Anales del Sistema Sanitario de Navarra. 2008;31:105-26.

4. Cheng A, Owens D. Republished: Marfan syndrome, inherited aortopathies and exercise: What is the right answer? Postgraduate medical journal. 2016;92(1083):51-6.

5. Imrie J, Heptinstall L, Knight S, Strong K. Observational cohort study of the natural history of Niemann-Pick disease type C in the UK: a 5-year update from the UK clinical database. BMC neurology. 2015;15(1):257.

6. Villegas JB, Obando FS. La carga de la enfermedad genética en Colombia, 1996-2025.

7. Acosta-Guio J, Zarante IM. Consulta de genética médica en un hospital de segundo nivel en Colombia: impacto médico y social1. Revista Salud Bosque volumen. 2011;1(1):25-32.

8. Malambo D, Gómez D, Veloza L, Arbeláez J, Gómez C. Algunos aspectos hereditarios y ambientales en casos de fibrosis quística en la ciudad de Cartagena (Colombia).

9. Pizarro, D y Fundación Hernán Echeverría Olózaga. 2008. Estudio Socioeconomico de la población de Santa Ana, Barú.

10. Fundación Puerto Bahía y Fundación Mamonal. 2009. Diagnostico Socioeconómico de la Vereda Ararca, Unidad Comunera de Gobierno Rural de Santa Ana.

11. Puerto Bahía. Estudio del impacto ambiental: Capítulo 3, caracterización del medio socioeconomico. 2011; 1-122.

12. Santoyo, C. Anguerra, T. El hacinamiento como contexto: Estrategias metodológicas para su analisis. Revista Psicothema. 1992; 4 (2): 551-569.

13. Instituto de Investigación sobre Crecimiento y Desarrollo. Curvas y tablas de crecimiento. 2-36.

14. Netscher DT. Congenital hand problems. Terminology, etiology, and management. Clinics in plastic surgery. $1998 ; 25(4): 537-52$.

15. Morovic I. CG. Cirugía de mano en pediatría. Revista chilena de pediatría. 2005;76:86-90.

16. Vidal Ruiz CA, Pérez Salazar-Marina D, Calzada Vázquez-Vela C, Castañeda Leedor P. Anomalías congénitas más comunes de la mano. Rev Mex Ortopedia Pediátrica. 2012;14(1):5-11.

17. Porto Álvarez RF, Álvarez Núñez R, Porto Álvarez GM. Sindactilia compleja de la mano, un reto difícil. Presentación de dos casos. Revista Médica Electrónica. 2013;35:411-4419.

18. Mills JK, Wheeler L, Oishi SN. A case of familial syndactyly associated with eye and dental abnormalities. JAAPA : official journal of the American Academy of Physician Assistants. 2015;28(12):40-3.

19. Luo JY, Fu CH, Yao KB, Hu RS, Du QY, Liu ZY. [A case-control study on genetic and environmental factors regarding polydactyly and syndactyly]. Zhonghua liu xing bing xue za zhi = Zhonghua liuxingbingxue zazhi. 2009;30(9):903-6.

20. Reig G, Concha ML. Impronta genómica y desarrollo embrionario. International Journal of Morphology. 2012;30:1453-7.

21. Moreno, M. Barreiro, E. Impronta genómica. An Esp Pediatr. 1998; 48: 567-574.

22. Armineta, E. Enfermedades ligadas al cromosoma $X$ sexual, Boletin Medico-Facultad de Medicina UAS. Bol Med. 2004; 5: 20-24. 